\title{
Characterizing the adult and larval transcriptome of the multicolored Asian lady beetle, Harmonia axyridis
}

Lindsay A Havens, Matthew D MacManes

The reasons for the evolution and maintenance of striking visual phenotypes are as widespread as the species that display these phenotypes. While study systems such as Heliconius and Dendrobatidae have been well characterized and provide critical information about the evolution of these traits, a breadth of new study systems, in which the phenotype of interest can be easily manipulated and quantified, are essential for gaining a more general understanding of these specific evolutionary processes. One such model is the multicolored Asian lady beetle, Harmonia axyridis, which displays significant elytral spot and color polymorphism. Using transcriptome data from two life stages, adult and larva, we characterize the transcriptome, thereby laying a foundation for further analysis and identification of the genes responsible for the continual maintenance of spot variation in $\mathrm{H}$. axyridis. 
1 Characterizing the Adult and Larval Transcriptome of the Multicolored Asian Lady Beetle,

2 Harmonia axyridis

3

4 Lindsay Havens ${ }^{1^{*}}$ and Matthew MacManes ${ }^{1+}$

5

6

7 1. University of New Hampshire Department of Molecular Cellular and Biomedical Science

8 *lao95@wildcats.unh.edu

$9+$ matthew.macmanes@unh.edu

\section{Abstract:}

14 The reasons for the evolution and maintenance of striking visual phenotypes are as widespread as the species that display these phenotypes. While study systems such as Heliconius, the neotropic butterfly, and Dendrobatidae, poison dart frogs, have been well characterized and provide critical information about the evolution of these traits, a breadth of new study systems, in which the phenotype of interest can be easily manipulated and quantified, are essential for gaining a more general understanding of these specific evolutionary processes. One such model is the multicolored Asian lady beetle, Harmonia axyridis, which displays significant elytral spot and color polymorphism. Using transcriptome data from two life stages, adult and larva, we characterize the transcriptome, thereby laying a foundation for further analysis and identification of the genes responsible for the continual maintenance of spot variation in H. axyridis. 


\section{Introduction:}

33 The evolution and maintenance of phenotypic polymorphism and striking visual phenotypes have 34 fascinated scientists for many years (Darwin, 1859; Endler, 1986; Fisher, 1930; Gray and McKinnon, 35 2006; Joron et al., 2006). In general, insects have become increasingly popular as study organisms to examine phenotypic variation (Jennings, 2011; Joron et al., 2006). One such insect displaying extensive elytra and spot variation that has yet to be extensively studied is the Asian Multicolored Ladybeetle, Harmonia axyridis.

The mechanisms responsible for the evolution of these phenotypes are as widespread as the species that display them. Aposematism, crypsis, and mimicry may play a role in the evolution of phenotypic variation in the animal kingdom. Members of family Dendrobatidae, poison dart frogs, are aposematically colored (Cadwell, 1996), while Tetrix subulata grasshoppers maintain their phenotypic polymorphism to aid in crypsis (Karpestam et al., 2014). A mimicry strategy is utilized by one particularly well-characterized species that exhibits phenotypic polymorphism, the Neotropic butterfly system, Heliconius. The color, pattern, and eyespot polymorphism seen in Heliconius is thought to have arose as a result of Müllerian mimicry (Flanagan et al., 2004) and the supergenes underlying these traits have been well characterized (Kronforst et al., 2006, Joron et al., 2006, Jones et al., 2012).

These studies, aiming to elucidate the mechanistic links between phenotype and genotype, present a unique opportunity to gain insight into the inner workings of many important evolutionary processes. While systems like poison frogs and butterflies have been pioneering, the use of novel models, especially those that can be easily manipulated, are needed. One such study system that possesses many of the benefits of classical models, while offering several key benefits, described below, is the multicolored Asian lady beetle, Harmonia axyridis. Harmonia, which is common throughout North America, and easily bred in laboratory environments, possesses significant variation in elytral spot number and color.

Elytra color can be red, orange, yellow, or black and spot numbers of $H$. axyridis range from zero to twenty-two (Havens, personal observation). The patterning is symmetrical on both wings. In some animals, there is a center spot beneath the pronotum which leads to an odd number of spots. The elytral spots are formed by the production of melanin pigments (Bezzerides et al., 2007). The frequency of different morphs varies with location and temperature (Michie et al., 2010). The melanic morph is more prevalent in Asia when compared to North America (LaMana and Miller, 1996; Dobzhansky, 1933). A decrease in melanic $H$. axyridis has been shown to be correlated with an increase in average yearly temperatures in the Netherlands (Brakefield and de Jong, 2011). 
68 Sexual selection may play a role in color variation in H. axyridis. Osawa and Nishida (1992) remarked

69 that female $H$. axyridis might choose their mates based on melanin concentration. Their choice, 70 however, has been shown to vary based on season and temperature. Non-melanic (red, orange, or 71 yellow with any spot number) males have a higher frequency of mating in the spring-time, while melanic 72 (black) males have an increased frequency of mating in the summer. While this has been shown with 73 respect to elytral color, no such findings have occurred for spot number. Although these spot patterns 74 are believed to be related to predator avoidance, thermotolerance, or mate choice (Osawa and Nishida, 75 1992), the genetics underlying these patterns is currently unknown.

To begin to understand the genomics of elytral coloration and spot patterning, we sequenced the 78 transcriptome of a late-stage larva and adult ladybug. These results lay the groundwork for future study of the genomic architecture of pigment placement and development in $H$. axyridis.

Methods and Materials:

One larval (Figure 1a) and one adult (Figure 1b) H. axyridis were captured on the University of New Hampshire campus in Durham, New Hampshire $\left(43.1339^{\circ} \mathrm{N}, 70.9264^{\circ} \mathrm{W}\right)$. The adult was orange with 18 spots. The insects were placed in RNAlater and immediately stored in a $-80 \mathrm{C}$ freezer until RNA extraction was performed. The RNA from both individuals was extracted following the TRIzol extraction protocol (Invitrogen, Carlsbad USA). The entire insect was used for the RNA extraction protocol. The quantity and quality of extracted RNA was analyzed using a Qubit (Life Technology, Carlsbad USA) as well as a Tapestation 2200 (Agilent technologies, Palo Alto USA) prior to library construction. Following verification, RNA libraries were constructed for both samples following the TruSeq stranded RNA prep kit (Illumina, San Diego USA), which includes PolyA purification of mRNA. To allow multiple samples to be run in one lane, a unique index code was added to each sample. These samples were then pooled in equimolar quantities using Tapestation analysis. The multiplexed libraries were then sent to the New York Genome Center (New York, USA) for sequencing on a single lane (125bp paired end) of the HiSeq 2500 sequencer. 
98

99

100

101

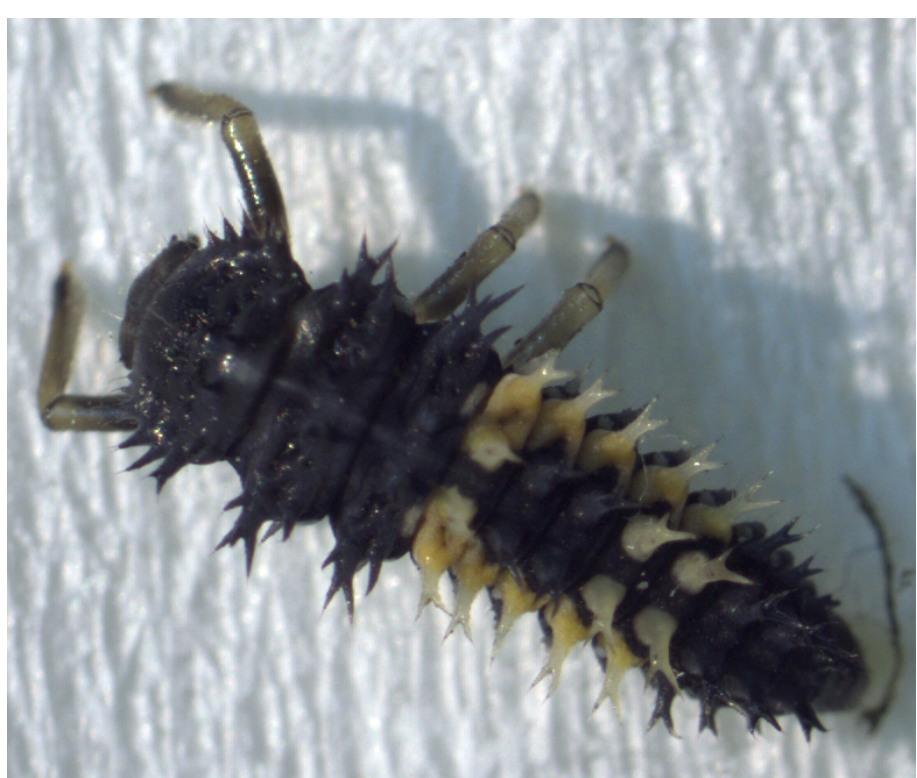

Figure 1a: The larva used for transcriptome sequencing.

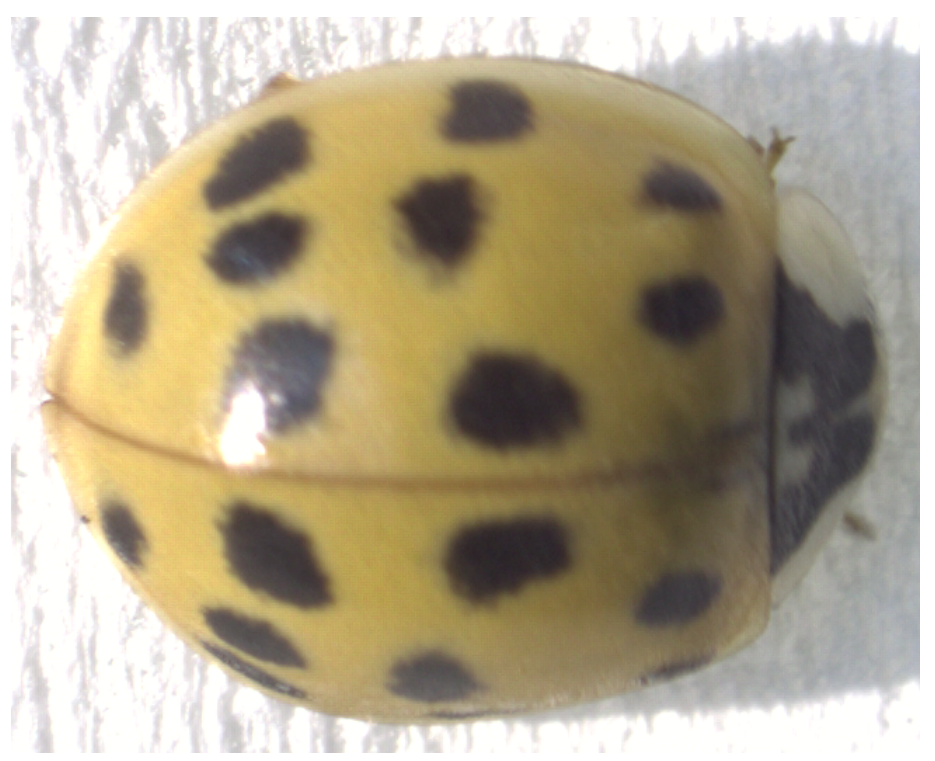

Figure 1b: The adult used for transcriptome sequencing.

\section{Sequence Data Preprocessing and Assembly}

The raw sequence reads corresponding to the two tissue types were error corrected using the software BLESS (Heo et al., 2014) version 0.17 (https://goo.gl/YHxlzl, https://goo.gl/vBh7Pg). The errorcorrected sequence reads were adapter and quality trimmed following recommendations from MacManes (MacManes, 2014) and Mbandi (Mbandi, 2014). Specifically, adapter sequence contamination and low quality nucleotides (defined as Phred<2) were removed using the program 
114 Trimmomatic version 0.32 (Bolger, 2014) called from within the Trinity assembler version 2.1.1 (Haas,

115 2013). Reads from each tissue were assembled together to created a joint assembly of adult and larva

116 transcripts using a Linux workstation with 64 cores and 1Tb RAM. We used flags to indicate the stranded

117 nature of sequencing reads and set the maximum allowable physical distance between read pairs to

118 999nt (https://goo.gl/ZYP08M).

120 The quality of the assembly was evaluated using transrate version 1.01 (Unna-Smith 2015;

121 (https://goo.gl/RpdQSU). Transrate generates quality statistics based on a process involving mapping 122 sequence reads back to the assembled transcripts. Transcripts supported by properly mapped reads of 123 a sufficient depth (amongst other things) are judged to be of high quality. In addition to generating quality 124 metrics, transrate produces an alternative assembly with poorly-supported transcripts removed. This 125 improved assembly was used for all downstream analyses and QC procedures. We then evaluated 126 transcriptome completeness via use of the software package BUSCO version 1.1b (Simão 2015). 127 BUSCO searches against a database of highly-conserved single-copy genes in Arthropoda 128 (https://goo.gl/bhTNdr). High quality, complete transcriptomes are hypothesized to contain the vast 129 majority of these conserved genes as they are present in most other species.

To remove assembly artifacts remaining after transrate optimization, we estimated transcript abundance

132 using 2 software packages - Salmon version 0.51 (Patro 2015; https://goo.gl/01UIF6) and Kallisto 133 version 0.42.4 (Bray, 2015; https://goo.gl/BsQMpr). Transcripts whose abundance exceeded 0.5 TPM 134 in either adult or larval datasets using either estimation method were retained. TPM is different from 135 FPKM with regards to the order of operations performed, as TPM normalizes for gene length first. We 136 evaluated transcriptome completeness and quality, again, after TPM filtration, using BUSCO and 137 transrate, to ensure that our filtration processes did not significantly effect the biological content of the 138 assembly.

140 We identified and removed potential plant, fungal, bacterial and vertebrate contamination by using a 141 blastx search. We created a custom protein database based on the collection of protein sequences 142 from each taxonomic group available for download from RefSeq 143 (ftp://ftp.ncbi.nlm.nih.gov/refseq/release/). We queried this database, inferring a given sequence to be 144 a contaminate if the best blast (= lowest e-value) was plant, fungal, bacterial or vertebrate on origin, 145 rather than invertebrate.

\section{Assembled Sequence Annotation}

The filtered assemblies were annotated using the software package dammit 
151 process, which involves use of blast (Camacho, 2009), TransDecoder (version 2.0.1,

$152 \mathrm{http}: / /$ transdecoder.github.io/), and hmmer version 3.1b1 (Wheeler 2013). In addition to this, putative

153 secretory proteins were identified using the software signalP, version 4.1c (https://goo.gl/FaOQSj).

155 In addition to this, we attempted to identify loci involved in color, color patterning, and more generally 156 phenotypic polymorphism. To accomplish this, we downloaded a set of 1008 candidate genes previously identified as underlying phenotypic evolution in other species, from Dryad (Martin and

Orgogozo, 2013a and 2013b). We used the gene name listed in this dataset to download the protein sequence from Uniref90. We used a blastX search strategy to identify potential homologues in the dataset.

To identify patterns of gene expression unique to each life stage, we used the expression data as per above. We identified transcripts expressed in one stage but not the other, and cases where expression occurred in both life stages. The Uniprot ID was identified for each of these transcripts using a blastX search (https://goo.gl/J9saMi), and these terms were used in the web interface Amigo (Carbon et al., 2009) to identify Gene Ontology terms that were enriched in either adult or larva relative to the background patterns of expression. The number of unique genes contained in the joint assembly was calculated via a BLAST search of the complete gene sets of human, Homo sapiens, fruit fly, Drosophila melanogaster, and the flour beetle, Tribolium casteneda.

Results and Discussion:

\section{Data Availability}

All read data are available under ENA accession number PRJEB13023. Assemblies and data matrices are available at https://goo.gl/D3xh65, and will be moved to Dryad following manuscript acceptance.

\section{RNA extraction, Assembly and Evaluation}

179 RNA was extracted from whole bodies of both the adult and the larva stage of a single Harmonia 180 axyridis. The quality was verified using a Tapestation 2200 (all RIN scores > 8) as well as a Qubit. The 181 initial concentration for the larva sample was $83.2 n g^{*} \mu \mathrm{L}-1$, while the initial concentration for the adult 182 sample was $74.7 \mathrm{ng}^{*} \mu \mathrm{L}-1$. The number of strand-specific paired end reads contained in the adult and 183 larva libraries were 58 million and 67 million, respectively. The reads were 125 base pairs in length. 
185 The raw Trinity assembly of the larval and adult reads resulted in a total of 171,117 contigs (82Mb) 186 exceeding 200nt in length. 526 contaminate sequences were removed. This assembly was evaluated 187 using Transrate, producing an initial score of 0.10543 , and and optimized score of 0.29729. The 188 optimized score indicated that the optimized assembly was better than $50 \%$ of NCBI-published de novo 189 transcriptomes (Unna-Smith 2015). This transrate optimized assembly (89,305 transcripts, 62Mb) was 190 further filtered by removing transcripts whose expression was less than 0.5 TPM. After filtration, 33,648 191 transcripts $(44 \mathrm{Mb})$ remained. To assess for the inadvertent loss of valid transcripts, we ran BUSCO 192 before and after this filtration procedure. The percent of Arthropoda BUSCO's missing from the 193 assembly rose slightly, from $18 \%$ to $21 \%$. Transrate was run once again, and resulted in a final 194 assembly score of 0.29112 . This score is indicative of a high-quality transcriptome appropriate for 195 further study (Unna-Smith 2015). In an attempt at understanding how many distinct genes our 196 transcriptome contained, we conducted a blast search against Homo sapiens, Drosophila 197 melanogaster, and Tribolium casteneda. This search resulted in 7,246, 7,739, and 7,741 matches 198 unique to Harmonia axyridis, which serve as estimates of the number of unique genes expressed in 199 these two life stages. The final assembly is available at https://goo.gl/nWdBuv

\section{Annotation}

The assembled transcripts were annotated using the software package dammit!, which provided annotations for 23,304 , or $69 \%$ of the transcripts (available here: https://goo.gl/gpGXLG). These annotations included putative protein and nucleotide matches, 5- and 3-prime UTRs, as well as start and stop codons. In addition to this, analysis with Transdecoder yielded 14,518 putative protein sequences (available here: https://goo.gl/qVLWwD), which were annotated by 4,139 distinct Pfam protein families, while 176 transcripts were determined to be non-coding (ncRNA) based on significant matches to the Rfam database (available here: https://goo.gl/x1n7jC). Lastly, 2,925 proteins (7.8\% of total) were determined to to be secretory in nature by the software package signalP (available here: https://goo.gl/z0ra1g).

Annotation of the sequence dataset resulted in the identification of host of transcripts that may be of interest to other researchers including: 43 heat-shock and 8 cold-shock transcripts, 87 homoeboxdomain containing transcripts, 122 7-transmembrane-containing (18 GPCR's) transcripts, 13 solute carriers, 143 ABC-transport-containing transcripts, and 21 OD-S (pheromone-binding) transcripts.

218 A complement of immune-related genes were discovered as well. These include a single member of 219 the Attacins and Coleoptericins, two TLR-like genes, seven Group 1, and 34 Group 2 C-type lectin 
220 Receptors (CLRs). Two CARD-containing Cytoplasmic pattern recognition receptor (CRR) genes were

221 discovered, as were 3 MAP kinase containing transcripts. Finally, 119 RIG-I-like receptors (RLR) were

222 found.

223

224 The focused search for genes previously implicated in phenotypic polymorphism (Martin and Orgogozo, $2252013 a$ and 2013b) identified 483 Harmonia transcripts, corresponding to 65 distinct loci (Supplementary 226 table 1). These loci include the transcription factors bab1, Distal-less, Optix, enzymes BCMO1, ebony, 227 yellow, and transporters ABCC2, SLC24A5, and TPCN2, all related to color and color patterning. These 228 genes, and the others identified in Supplementary table to are likely to provide fodder for future 229 research.

230

231 Gene expression was estimated for each transcript for both adult and larva (Figure 2, data available 232 here: https://goo.gl/wM3TV7). For all transcripts expressed in the adult, the mean TPM=29.7 (max= $23380,016.9, S D=500.8)$ while the mean larval TPM=29.71 (max=166,264, SD=941). When analyzing 234 transcripts found uniquely in these two tissues, mean adult TMP=9.9 ( $\max =3,037, \mathrm{SD}=86)$ and for larva $235 \mathrm{TPM}=8.6(\max =687, \mathrm{SD}=41)$.

1922 transcripts expressed in

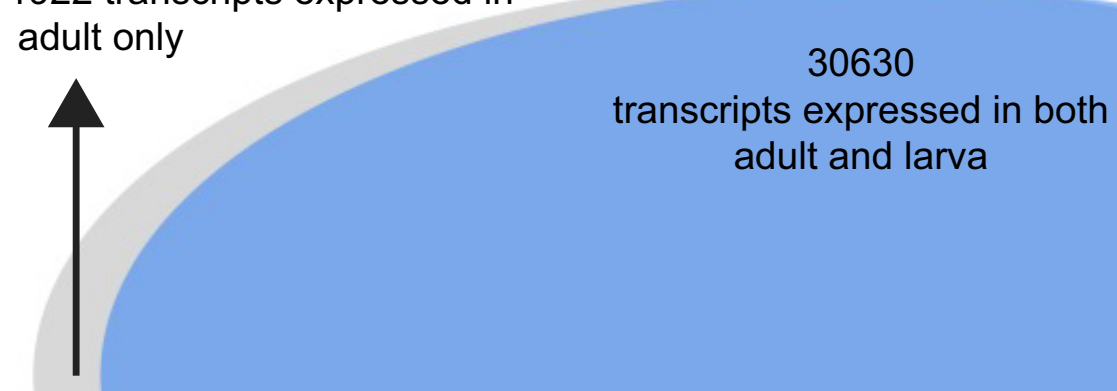

Figure 2: The Venn diagram representing the number of transcripts expressed in both adult and larva, as well as those expressed uniquely in one or the other.

Analysis of the differences between adult and larval life stages were carried out as well. Because these

242 life stages were only sequenced with a single individual each, they should be interpreted with some 
243 caution. The vast majority of transcripts were observed in both life stages $(n=30,630,91 \%)$, with a small

244 number being expressed uniquely in larva $(n=1,094)$ and adult $(n=1,922)$. Of these transcripts

245 expressed uniquely in either larva or adult, $45 \%$ and $42 \% \mathrm{c}$ were annotated using at least one method

246 via the software package dammit. $6.1 \%$ and $4.6 \%$, respectively, were found to be secretory in nature

247 via signalP analysis.

248

249 Conclusions:

250 Phenotypic polymorphisms and striking visual phenotypes have fascinated scientists for many years.

251 The breadth of evolutionary causes for the maintenance of these phenotypes are as numerous as the

252 species that display them. One organism, Harmonia axyridis, provides a unique opportunity to explore

253 the genetic basis behind the maintenance of an easy to quantify variation - elytral spot number. While

254 understanding these genomic mechanisms is beyond the scope of this paper, we do provide a reference

255 transcriptome for $H$. axyridis, a foundational resource for this work.

256

257 This study indicates that most gene expressed at levels greater than .5 TPM were shared seen in both 258 the adult and larval individuals. While the majority of proteins identified in the assembled transcriptome 259 were structural in function, analyses of protein families using the Pfam database indicated the presence 260 of pigment proteins. In particular, RPE65, which functions in the cleavage of carotenoids, was found. In 261 H. axyridis, increased carotenoid pigmentation has been linked to increased alkaloid amounts (Britton 262 et al., 2008). In addition, the elytral coloration of the seven spot ladybug, Coccinella septempunctata, is 263 a result of several carotenoids (Britton et al., 2008). While larva are mostly black (Figure 1a), we posit 264 that the orange sections on the lower back could be due to carotenoid production. Moreover, this study 265 provides a necessary foundation for the continued study of the genetic link between genes and the 266 maintenance of variation in $H$. axyridis. 


\section{Literature Cited:}

270 Bezzerides A. L., McGraw K. J., Parker R. S., Husseini J. (2007). Elytral color as a signal of chemical defense in the Asian ladybird beetle Harmonia axyridis. Behavior Ecology and Sociobiology 61:

273

274

275

276

277

278

279

280

281

282

283

284

285

286

287

288

289

290

291

292

293

294

295

296

297

298

299

300

301 1401-1408.

Bolger A. M., Lohse M., Usadel., B. (2014). Trimmomatic: A flexible trimmer for Illumina Sequence Data. Bioinformatics, btu170.

Brakefield P. M., de Jong P. W. (2011). A steep cline in ladybird melanism has decayed over 25 years: a genetic response to climate change? Heredity 107: 574-578.

Bray N., Pimental H., Melsted P., Lior P. (2015). Near-optimal RNA-seq quantification. eprint arXiv:1505.02710

Britton G., Liassen-Jesen, S., Pfander H.(2008). Carotenoids, Vol. 4: Natural Functions. Springer. DIO: 0.1007/978-3-7643-7499-0

Cadwell J. P., (1996). The Evolution of myrmecophagy and its correlates in poison frogs (Family Dendrobatidae). Journal of zoology 240: 75-101.

Camacho C., Coulouris G., Avagyan V., Ma N., Papadopoulos J., Bealer K., Madden T. L. (2009). BLAST+: architecture and applications. BMC Bioinformatics doi: 10.1186/1471-2105-10-421.

Carbon S., Ireland A., Mungall C.J., Shu S., Marshall B., Lewis S. (2009). AmiGO Hub, Web Presence Working Group. AmiGO: online access to ontology and annotation data. Bioinformatics 25(2):288-9.

Darwin C. (1859). The Origin of Species. New York, New York: Bantam Dell.

Dobzhansky T. (1993). Geographical variation in ladybeetles. The American Naturalist 67: 97-126.

Endler J. A. (1986). Natural Selection in the Wild. Princeton, New Jersey: Princeton University Press.

Fisher, R. A. (1930). The Genetical Theory of Natural Selection. Oxford: Clarendon Press.

Flanagan N.S., Tobler A., Davison A., Pybus O.G., Kapan D. D., Planas S., Linares M., Heckel D., McMillan W. O. (2004). Historical demography of Müllerian mimicry in the neotropical Heliconius butterflies. PNAS 101: 9704-9709.

Gray S. M., McKinnon J. S. (2006). Linking color polymorphism maintenance and speciation. ScienceDirect: doi:10.1016/j.tree.2006.10.005

Haas B. J., Papanicolaou A., Yassour M., Grabherr M., Blood P. D., Bowden J., Couger M. B., Eccles D., Li B., Lieber M., MacManes M. D., Ott M., Orvis J., Pochet N., Strozzi F., Weeks N., Wasterman R., William T., Dewey C. N., Henschel R., LeDuc R. D., Friedman N., Regev A. (2013). De novo transcript sequence reconstruction from RNA-seq using Trinity platform for reference generation an analysis. Nature Protocols 8: 1494-1512. 
302 Heo Y., Wu X., Chen D., Ma J., H W. (2013). BLESS: Bloom filtered-based error correction solution for high-throughput sequencing reads. Bioinformatics doi: 10.1093/bioinformatics/btu030.

304

305

306

307

308

309

310

311

312

313

314

315

316

317

318

319

320

321

322

323

324

325

326

327

328

329

330

331

332

333

334

Jennings B. H. (2011). Drosophila - a versatile model in biology \& medicine. MaterialsToday 14: 190195.

Jones R.T., Salazar P. A., ffrench-Constant R. H., Jiggins C.D., Joron M. (2012). Evolution of a mimicry supergene from a multilocus architecture. Proc. R. Soc. B 279: doi: 10.1098/rspb.2011.0882.

Joron M., Jiggins C. D., Papanicolaou A., McMillan W. O. (2006). Heliconius wing patterns: an evodevo model for understanding phenotypic diversity. Heredity 97: 157-167.

Karpestam E., Merilaita S., Forsman A. (2014). Natural levels of colour polymorphism reduce performance of visual predators searching for camouflaged prey. Biol J Linnean Soc. doi: 10.1111/bij. 12276 .

Kronforst M. R., Young L. G., Kapan D. D., McNeely C., O'Neill R. J., Gilbert L. E. (2006). Linkage of butterfly mate preference and wing color preference cue at the genomic location of wingless. PNAS 103: 6575-6580.

LaMana M.L., Miller J.C. (1996). Field observations on Harmonia axyridis Pallas (Coleoptera: Coccinellidae) in Oregon. Biological Control 6: 232-237.

MacManes M. D. (2014). On the optimal trimming of high-throughput mRNA sequence data. Front Genet 5: 13. doi: 10.3389/fgene.2014.00013.

Mbandi S. K., Hesse U., Rees J. G., Christoffels A. (2014). A glance at quality score: implication for de novo transcriptome reconstruction of Illumina reads. Frontiers in Genetics (5): doi: 10.3389/fgene.2014.00017.

Martin A, Orgogozo V (2013a) The loci of repeated evolution: a catalogue of genetic hotspots of phenotypic variation. Evolution 67(5): 1235-1250. http://dx.doi.org/10.1111/evo.12081

Martin A, Orgogozo V (2013b) Data from: The loci of repeated evolution: a catalogue of genetic hotspots of phenotypic variation. Dryad Digital Repository. http://dx.doi.org/10.5061/dryad.v66p0

Michie L.J., Mallard F., Majerus M.E.N., Jiggins F.M. (2010). Melanic through nature or nurture: genetic polymorphism and phenotypic plasticity in Harmonia axyridis. Journal of Evolutionary Biology 23:1699-1707.

Osawa N., Nishida T., (1992). Seasonal variation in elytral colour polymorphism in Harmonia axyridis (the ladybird beetle): the role of non-random mating. Heredity 69: 297-307.

Patro R., Suggal G.,Kingsford C. (2015). Accurate, fast, and model-aware transcript expression quantification with Salmon. bioRxiv: doi: http://dx.doi.org/10.1101/021592 
335 Simão F. A., Waterhouse R. M., loannidis P., Kriventseva E. V., Zdobnov E. M. (2015). BUSCO: assessing genome assembly and annotation completeness with single-copy orthologs. Bioinformatics dio: 10.1093/bioinformatics/btv351.

338 Smith-Unna R. D., Boursnell C., Patro R., Hibberd J. M., Kelly S. (2015). TransRate: reference free quality assessment of de-novo transcriptome assemblies. bioRxiv: dio http://dx.doi.org/10.1101/021626.

341 Wheeler T. J., Eddy S. R. (2013). nhmmer:DNA homology search with profile HMMs. (2013).

342 Bioinformatics: doi: 10.1093/bioinformatics/btt403 